\title{
Context excitation and modulation of conditioned sexual behavior
}

\author{
CHANA K. AKINS \\ University of Kentucky, Lexington, Kentucky
}

\begin{abstract}
Three experiments were conducted to investigate direct and modulatory influences of context in the conditioned sexual behavior of male Japanese quail. A preference test procedure was used to assess the acquisition of contextual excitation. In Experiment 1, following direct context-unconditioned stimulus (US) pairings, male quail shifted their contextual preference from an initially preferred context to one in which they received copulatory opportunity with a female quail (US). Unpaired control group subjects did not demonstrate this shift in preference. This place preference procedure was used in Experiments 2 and 3 to assess contextual excitation when context was trained in the presence of a discrete conditioned stimulus (CS). Experiment 2 provided evidence that context can modulate responding to a discrete CS. In Experiment 3, we varied the spatial contiguity between the context and the US. Some subjects received the US directly in the training context, whereas other subjects received the US in an alternate context. Contextual excitation was evident only in subjects that received the former. Thus, there is a dissociation between the modulatory and excitatory properties of context in sexual conditioning that may depend on the context-US spatial contiguity.
\end{abstract}

Previous research involving unsignaled presentations of an unconditioned stimulus (US) in an environmental context suggests that contextual cues may serve as a reliable predictor of subsequent reinforcement (e.g., Fanselow, 1990; Zamble, Hadad, Mitchell, \& Cutmore, 1985). After several presentations of a US in the context, various features of the context may become associated with the US and, via Pavlovian conditioning, elicit a change in behavior. Contextual conditioning that involves the formation of a context-US association in the absence of a conditioned stimulus (CS) has been demonstrated with USs such as food, electric shock, and exposure to potential sexual partners (see, e.g., Bolles \& Collier, 1976; Colwill, Absher, \& Roberts, 1988; Papini, Mustaca, Tiscornia, \& DiTella, 1987; Rescorla, Durlach, \& Grau, 1985; Roald Maes \& Vossen, 1993; Zamble et al., 1985). Quantitative changes in behavior, such as increased locomotor activity, and context preferences seem to be appropriate indicators of contextual excitation. Thus, it is evident that context may serve as a CS and function as a reliable predicter of US reinforcement.

In the presence of a discrete CS, context may serve to "set the occasion" for the CS-US association. The occasionsetting function is based on Holland's analysis of positive and negative feature discriminations (Holland, 1983,

This research was supported by National Science Foundation Grant IBN-9511656 C.K.A. I gratefully acknowledge Rick Bevins, Michael Bardo, Michael Domjan, and Thomas Zentall for their helpful comments on earlier versions of this manuscript, and Charles T. Collier for help in collecting data. Thanks also to Ralph Miller and anonymous reviewers for their comments on the manuscript. Send correspondence to: C. K. Akins, Department of Psychology, University of Kentucky, Lexington, KY 40506-0044 (e-mail: ckakin1@pop.uky.edu).
1992). In a feature-positive discrimination, a target $A$ is accompanied by a feature stimulus $\mathrm{X}$ during reinforced trials and not accompanied by $\mathrm{X}$ during nonreinforced trials $(\mathrm{XA}+, \mathrm{A})$. Conversely, in a feature-negative discrimination, target stimulus $A$ is reinforced alone but not in the presence of the feature stimulus $(\mathrm{A}+, \mathrm{XA}-)$. Within this framework, the feature $X$ should acquire the ability to indicate when $A$ will be reinforced and nonreinforced, rather than simply signaling when the US will or will not occur.

Feature-positive and feature-negative discriminations have also been referred to as positive and negative modulators. Modulatory mechanisms of discrete stimuli have been well studied (for a review, see Swartzentruber, 1995). The available evidence of contextual control suggests that contexts may come to control responding via mechanisms similar to the modulatory mechanisms of discrete conditioned stimuli (e.g., Balaz, Capra, Hartl, \& Miller, 1981; Bouton, 1984; Bouton \& King, 1983; Bouton \& Swartzentruber, 1986; Grahame, Hallam, Geier, \& Miller, 1990; Rescorla et al., 1985; Swartzentruber, 1991; Swartzentruber \& Bouton, 1988). Furthermore, just as discrete stimuli serve as modulators, contexts appear to control responding to a target stimulus independently of their association with a US. Using a conditioned suppression preparation with rats, Bouton and Swartzentruber (1986) failed to find evidence for contextual excitation and inhibition after explicitly training context to set the occasion for the presence or absence of shock. Also, repeated extinction exposures of a context explicitly trained as an occasion setter did not affect the context's ability to modulate responding to the CS. In another conditioned suppression experiment, Bouton and King (1986) used preference tests to examine the effects of discrete stimuli in 
a context that had been made excitatory by pairing it with a US. Only stimuli that had been conditioned and were undergoing extinction were affected by contextual excitation. More recently, Swartzentruber (1991) found that a light stimulus that was trained to set the occasion for food signaled by a tone CS blocked contextual control of responding to the tone. Conversely, a context that signaled reinforcement of the tone blocked acquisition of modulatory control by the light. Thus, the ability of a context to modulate CS performance may be relatively independent of its direct association with the US, and contextual excitation may not be necessary for contextual control of a discrete CS.

Modulatory effects of context have also been identified in the sexual behavior system. Domjan (1994) formulated a behavior system for sexual conditioning in which he proposed that sexually conditioned contextual cues influenced sexual responses primarily by modulating responses elicited by localized types of stimuli. This model of sexual conditioning is based on previous research done with Japanese quail as subjects. Domjan, Greene, and North (1989) tested the modulatory role of context on species-specific cues of female quail by using a taxidermically prepared model of the head and neck. One group of subjects was allowed to copulate with a live female quail in the same context in which they were later tested with the head and neck model. A second group of subjects was provided with copulatory opportunities with a live female in an alternate context. The group that received the head and neck model in the same context as that in which copulatory opportunity with a live female took place responded with a higher frequency of copulatory behaviors than did the other group. These results indicate that conditioned contextual cues modulate responding of sexual behavior by increasing the effectiveness of female quail features in eliciting copulatory behavior.

More recently, Hilliard, Nguyen, and Domjan (1997) conducted a powerful demonstration of how context comes to control the effectiveness of species-specific female cues in eliciting an appetitive component of the copulatory behavior sequence: approach behavior. In their experiment, male quail were given a one-trial exposure to a context followed by copulatory opportunity with a female quail. A control group received context and copulatory opportunity in an unpaired fashion. Subjects were tested by reintroducing them to the context in the presence of a taxidermic head and neck model of a female. Male quail that had received sexual experience with a live female in the context demonstrated more sexual approach behavior toward the taxidermic head model than did the control group. Moreover, the effect occurred with intervals of up to $4 \mathrm{~min}$ between the onset of context presentation and the introduction of the female. These findings provide evidence for one-trial modulatory effects of context in the sexual conditioning.

Yet although there is substantive evidence for modulatory effects of context in sexual conditioning, the mech- anisms of contextual control of conditioned sexual behavior have not been investigated. The present research contributes a novel experimental procedure with which to begin to elucidate the modulatory and excitatory mechanisms of contextual control of conditioned sexual behavior. In Experiment 1, we assessed direct contextual excitation of sexually conditioned behavior in male quail by pairing a context with sexual opportunity with a female quail. Experiments 2 and 3 were conducted to investigate the contextual excitation and the modulatory influence of context on discrete CSs. Conditioned approach behavior was used as an index of the degree of control by the CS and contextual preference tests were used to assess the acquisition of contextual excitation.

\section{EXPERIMENT 1}

Zamble et al. (1985) have provided what little evidence there is for contextual excitation of sexual arousal via a direct context-US association, rather than a modulatory role of context on other stimuli. In their experiments, male rats were carried to a holding room and placed in a plastic tub with wood shavings (the putative contextual CS). After $10 \mathrm{~min}$ in the tubs, they were exposed to a receptive female rat (US) located behind a wire mesh screen. Subjects in a control group were given both CS and US presentations in a random order. Subjects that received context-US pairings showed a decrease in latency to ejaculate with a female after, as opposed to before, conditioning trials. In later experiments (Zamble, Mitchell, \& Findlay, 1986), however, the investigators failed to find within-group differences in ejaculation latency during context-US tests and during tests when the putative context was absent. Thus, although their results provide good evidence for contextual control of sexual arousal, it is not clear which cues were controlling conditioned responding in this paradigm.

The present experiment was designed to examine the direct association between context and a sexual US. Opportunity to copulate with a receptive female quail served as the US. Contextual excitation was measured in two ways. First, acquisition of context-US association was assessed in a context preference test procedure. A context preference test was conducted before any conditioning trials began and, subsequently, after every fifth conditioning trial. Context preference tests have been used to assess conditioned excitation in fear conditioning experiments (Bouton \& Bolles, 1985; Bouton \& King, 1983; Marlin, 1981; Odling-Smee, 1975, 1978) and appear to be a sensitive index of contextual excitation. Second, locomotor activity was recorded during each conditioning trial. Increased locomotor activity has been observed in pigeons in response to food conditioned contextual cues (Durlach, 1982). In addition, high levels of locomotor activity are evident with long CS-US intervals when a discrete CS is paired with copulatory opportunity (Akins, Domjan, \& Gutiérrez, 1994). To strengthen the case for conditioning, the extinction of context-US association 
was assessed in a similar manner as was acquisition, but with nonreinforced exposure to the context.

\section{Method}

\section{Subjects}

Japanese quail were hatched and raised in the laboratory from eggs obtained from a randomly bred colony maintained at the University of Kentucky. (These eggs originated from a stock of quail that were raised and housed at the University of Texas.) They were kept in mixed-sex groups in brooders. At 4-5 weeks of age, they were moved to individual wire mesh cages (GQF Manufacturing Co., Savannah, GA). Lights in the laboratory were on from 0600 to $2200 \mathrm{~h}$ daily, which provided sufficient photostimulation to maintain the birds in reproductive readiness. Food and water were available ad lib.

Subjects were selected for the experiment on the basis of a pretest for sexual behavior. A female bird was placed in the colony cage (home cage) of each male quail for $5 \mathrm{~min}$. Only subjects that copulated during the pretest were selected for the experiment. (Male quail are not likely to copulate with a female if they have not done so within $5 \mathrm{~min}$; Schein, Diamond, \& Carter, 1972.)

Twelve male Japanese quail (5-10 months old) served as subjects in the experiment. An equal number of female quail (5-10 months old) served during periods of copulatory opportunity. The assignment of females to males was rotated so that a given male never encountered the same female during successive training trials.

\begin{abstract}
Apparatus
The experimental procedures were conducted in six large test chambers $(182.9 \mathrm{~cm}$ wide $\times 30.5 \mathrm{~cm}$ high $\times 61 \mathrm{~cm}$ deep $)$, each of which consisted of two adjacent compartments separated by an opaque partition (see Akins et al., 1994, for illustration of similar apparatus). The two compartments of each test chamber were distinctly different. One compartment had sand spread over the floor, and orange-colored walls and ceiling-the sand context. The other compartment had a wire mesh floor, and green-colored walls and ceiling - the wire context.
\end{abstract}

\section{Procedure}

The subjects were tested in two squads. Each squad was housed in the test chambers during alternate 24 -h periods. While males of one squad were in the test chambers, the subjects of the other squad were in their home cages. The birds were exchanged at around noon each day. While in the test chambers, half of the subjects in each squad were housed in the sand context. The other half were housed in the wire context. This procedure was started approximately 14 days before any experimental manipulations, to habituate the birds to the test chambers and to the alternate housing procedure. The alternate housing has been used routinely in other quail sexual conditioning experiments (e.g., Domjan, O'Vary, \& Greene, 1988).

An initial 10 -min preference test was conducted prior to training, to assess the amount of time that subjects spent in each context. During the preference test, the partition between the two chambers was removed and the subjects were placed on a line marked off between the two compartments. All of the subjects demonstrated an initial preference for the context where they had been housed. Thus, the context in which the subjects spent the greatest amount of time was designated as the untrained context, the test chamber in which they were housed for the remainder of the experiment. The nonpreferred context was referred to as the trained context, where presentation of the US would occur during conditioning trials.

Subsequent 10 -min preference tests were conducted after every fifth conditioning trial (Bouton \& King, 1983; Mustaca, Gabelli, Papini, \& Balsam, 1991). During these tests, the partition between the two compartments was removed and the subjects were placed in the untrained context with access to the trained context.

Prior to conditioning, the subjects were randomly assigned to either Group Paired or Group Unpaired. During each conditioning trial, the subjects in Group Paired $(n=6)$ were placed in the trained context for $5 \mathrm{~min}$, at the end of which a female quail was placed in the chamber with them for a 5-min US period. The subjects in Group Unpaired ( $n=6$ ) were placed in their home cage $2 \mathrm{~h}$ before being placed into the trained context, and a quail hen was introduced there for a 5 -min US period. While Group Unpaired received US exposure in their home cage, Group Paired was given the same amount of time in the home cage. Thus, both groups received the same amount of exposure to the trained context. A total of 10 conditioning trials were conducted, 1 per day, each around mid-morning.

Following the last preference test of the acquisition phase, 10 extinction trials were conducted. The extinction trial procedures were similar to those for the conditioning trials, except that all of the subjects were given nonreinforced exposure to the trained context. As in the acquisition phase, a preference test was conducted after every 5 th trial.

\section{Response Measures}

The development of contextual conditioning was assessed in two ways. First, the amount of time that subjects spent in each context during each preference test was continuously recorded by one of the authors, using an event recorder program (prepared by James $\mathrm{Ha}$, Seattle). The event records were then used to calculate the percentage of time that each subject spent in the trained context. Second, the degree of pacing was monitored during each conditioning trial. To accomplish this, the floor of the trained context was marked in nine rectangles of equal size, and the frequency of line crossings was recorded during the trained context exposure, beginning immediately after the subjects were placed in the context. A line crossing was recorded when a subject crossed a line with both feet.

Half of the data for Preference Tests 1-3 were scored from videotapes by a second observer (the author or C.T.C.). The interobserver reliability correlation was .96 for the amount of time subjects spent in the trained context.

The .05 alpha level was used for all statistical decisions.

\section{Results and Discussion}

\section{Acquisition Phase}

Preference tests. The mean percentage of time that subjects spent in the trained context during each preference test for Group Paired and for Group Unpaired is shown in the top panel of Figure 1. Both groups spent little time in the trained context during the first preference test. However, after five context-US pairings in the trained context, Group Paired increased the amount of time that it spent in the context, whereas Group Unpaired showed little increase of time spent in the trained context. Similar levels of time spent in the trained context were evident during the third preference test (after five more conditioning trials). A two-factor analysis of variance (ANOVA) with group (paired vs. unpaired) and test (1-3) revealed significant main effects of group $[F(1,10)=36.25]$, and test $[F(2,20)=23.25]$, and a significant group $\times$ test interaction $[F(2,20)=13.78]$.

According to these results, male quail develop a preference for a context that has been paired repeatedly with copulatory opportunity. Initially, the subjects preferred 

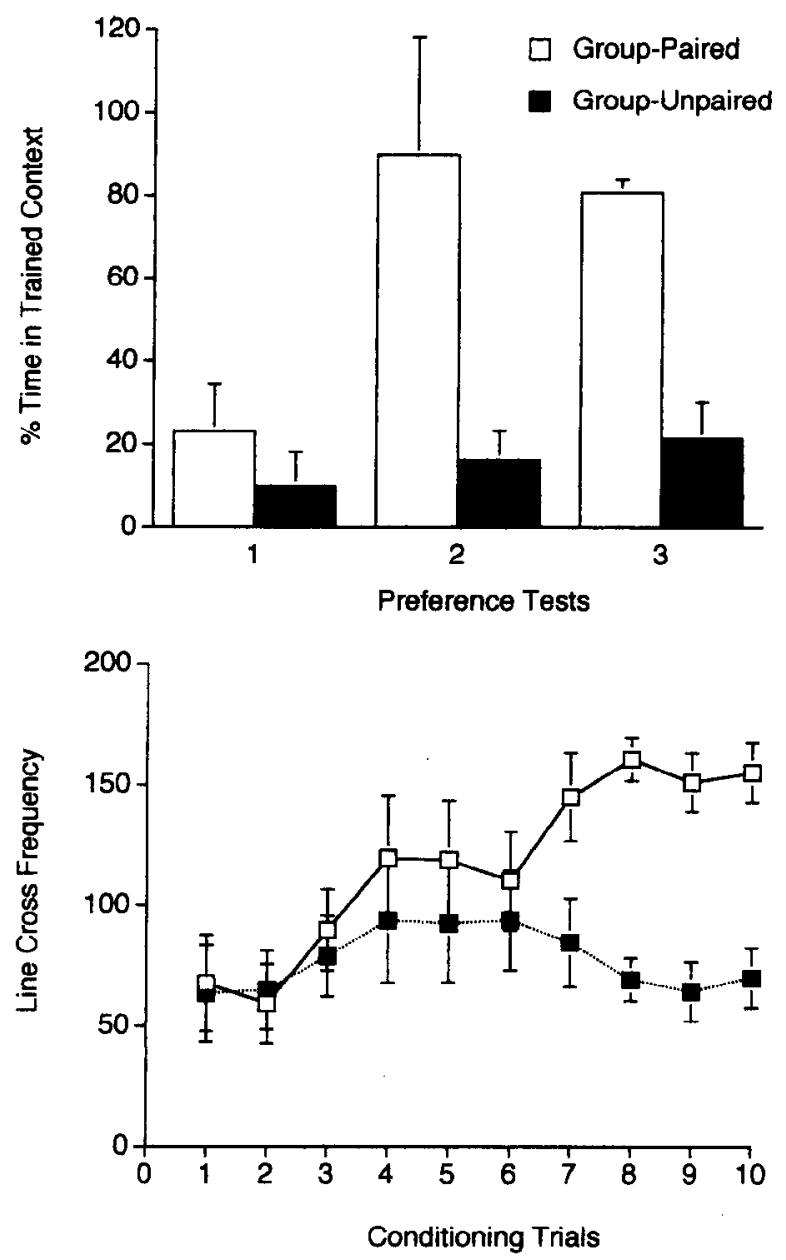

Figure 1. Mean $( \pm S E M)$ percentage of time that Group Paired and Group Unpaired spent in the trained context acmoss preference tests during acquisition (top panel) and their line cross frequency as a function of conditioning trials (bottom panel).

the context where they had been housed and had access to food and water. However, they later shifted their preference to the context where they were provided with access to a female quail. This shift in context preference was due to associative factors, because only subjects that received pairings of the context and the US increased their preference for the trained context. The control group that received an equal amount of copulatory opportunity and context exposure in an unpaired fashion did not increase its preference for the trained context.

Locomotor activity. The frequency of line crossings that paired and unpaired subjects made in the trained context during conditioning trials is summarized in the bottom panel of Figure 1. Group Paired increased its locomotor activity across conditioning trials, whereas Group Unpaired did not. Mean frequency of crosses ranged from 59.33 to 160.67 for Group Paired and from 63.5 to 93.83 for Group Unpaired. This resulted in a significant group $\times$ trial interaction $[F(9,90)=2.62]$. There was also a significant effect of group $[F(1,10)=6.7]$ and of trial $[F(9,90)=3.0]$. Thus, contextual excitation was also manifest as the acquisition of increased locomotor activity across conditioning trials for subjects that received context-US pairings but not for control group subjects. The increased locomotor activity that was observed in the paired group may represent a form of anticipatory behavior similar to the increased locomotor activity that occurs in food-conditioned contexts (Durlach, 1982) or that occurs in long-delay sexual conditioning experiments (Akins et al., 1994).

\section{Extinction Phase}

Preference tests. The percentage of time that subjects spent in the trained context during the preference tests of the extinction phase is shown in the top panel of Figure 2. After five context-no US trials, Group Paired decreased its time in the trained context, while Group Un-
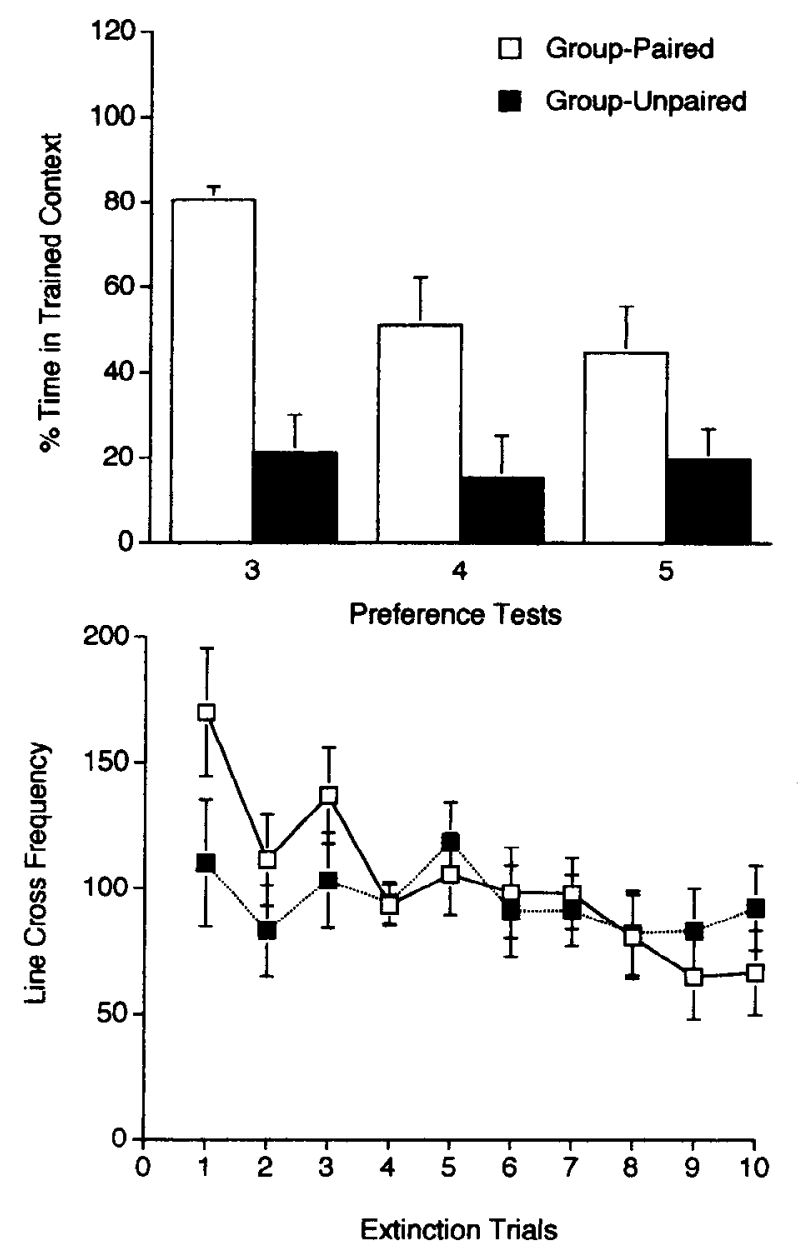

Figure 2. Mean $( \pm S E M)$ percentage of time that Group Paired and Group Unpaired spent in the trained context across preference tests during extinction (top panel) and their line cross frequency as a function of extinction trials (bottom panel). 
paired maintained a low level of preference for the trained context (Preference Test 4). A similar pattern was evident following five additional extinction trials (Preference Test 5). A two-factor ANOVA revealed a significant group $\times$ test interaction $[F(2,20)=3.91]$, a significant effect of group $[F(1,10)=14.62]$, and a significant main effect of test $[F(2,20)=5.74]$.

Locomotor activity. The bottom panel of Figure 2 depicts the frequency of line crosses Groups Paired and Unpaired made in the trained context across the $10 \mathrm{ex}-$ tinction trials. Group Paired decreased its frequency of line crosses as a function of extinction trials, whereas Group Unpaired did not. Analysis of these data resulted in a significant group $\times$ test interaction $[F(9,90)=2.2]$ and a significant effect of trial $[F(9,90)=5.22]$. There was no significant group effect $[F(1,10)=0.22]$.

Results from the extinction trials strengthen the case for associative learning. Group Paired decreased its preference for the trained context and showed a decrease in locomotor activity across extinction trials, whereas the unpaired control group did not.

\section{EXPERIMENT 2}

The findings in Experiment 1 provide support for the notion that context can acquire excitatory control of sexual conditioning through a direct association with a US. This is in accordance with associative theories of learning (e.g., Rescorla \& Wagner, 1972), which suggest that context and punctate cues are functionally equivalent and that they may acquire associative strength in a similar fashion.

There is also evidence that context may serve as a modulator for the acquisition of sexually conditioned responses to discrete stimuli. Domjan, Akins, and Vandergriff (1992) conducted an experiment that involved exposing male quail to two distinctive contexts that each contained a square holding box (the CS). Exposure to the two contexts differed only in that a female was released from the holding box in one context (the sexual context) but not in the other (the nonsexual context). Subjects increased the amount of time that they spent near the holding box across trials in the sexual context, whereas there was no change in time spent near the CS across trials in the nonsexual context. Several mechanisms may have been responsible for the contextual control of conditioned approach behavior made toward the box in the sexual context. One possibility is that context served as a modulator that provided information about when the holding box would release a female quail. Another possibility is that the conditioned excitatory properties of the context summated with the conditioned excitatory properties of the female holding box, resulting in more responding in the sexual context than in the nonsexual context. Because Domjan et al. (1992) did not test to determine whether or not the context acquired excitatory proper- ties, it is unclear which mechanism accounted for the role of context in their sexual conditioning experiment.

The present experiment was designed to replicate and extend the study by Domjan et al. (1992). The design was similar, except that the CS was a small wooden cylinder block and female quail were not released into the context. Rather, male quail received copulatory opportunity with a female bird (the US) after entering a side cage. During discrimination training, male quail were presented with the CS followed by the US in one context $(\mathrm{CXT}+)$ and a CS followed by no US in the other context $(\mathrm{CXT}-)$. Following discrimination training, subjects were given a context preference test between CXT + and $\mathrm{CXT}-$. A context preference test was given after discrimination training, to assess context excitation.

\section{Method}

\section{Subjects}

Twelve male Japanese quail served in the experiment. They were approximately 5-12 months old at the start of the experiment and had been raised under the same conditions as in Experiment 1. An equal number of female quail was used during the periods of copulatory opportunity. The assignment of females to males was rotated across trials as in Experiment 1.

\section{Apparatus}

The same six large test chambers were used as in Experiment 1. During habituation and conditioning trials, opaque partitions divided each large chamber into smaller compartments measuring $91.45 \mathrm{~cm}$ wide $\times 30.5 \mathrm{~cm}$ high $\times 61 \mathrm{~cm}$ deep. Adjacent compartments, separated by the partition, consisted of one of the two distinct contexts described in Experiment 1, either the sand context or the wire context. A smaller side cage that housed a female quail $(30.5 \mathrm{~cm}$ wide $\times 30.5 \mathrm{~cm}$ high $\times 61 \mathrm{~cm}$ deep) was attached at the end of each compartment. Between the male's test chamber and the female's side cage was a door. Approximately $13 \mathrm{~cm}$ in front of the door, a wooden cylinder-shaped white block $(2.54 \mathrm{~cm}$ in diameter) was suspended from the ceiling through a hook and was concealed by a black tube. Lowering the white block to the floor served as the CS. A small area $(28 \mathrm{~cm}$ wide $\times 23 \mathrm{~cm}$ deep) that contained the lowered block was marked off and referred to as the $C S$ zone. A string and pulley system was used to lower and raise the CS block and to open and close the door to the female's side cage. The strings for the CSs and doors were located several feet in front of the test chambers. Food and water were available ad lib along the wall of each compartment, opposite the CS area.

\section{Procedure}

Twelve subjects were assigned to two squads, and an alternate housing procedure was implemented. While males of one squad were housed in the test chambers, males of the other squad were housed in their colony cages (where they had been raised). The birds were exchanged around noon each day, with the squad that was in the test chambers moved to the colony cages and vice versa. This alternating housing procedure started approximately 14 days before any experimental treatment to habituate the birds to the test chambers. A female quail was housed in each of the side cages at all times throughout the experiment (except during CXT - trials) so that any noise she made in the side cage would not become a cue for impending sexual opportunity. The habituation of the birds to the test cages has been conducted in other experiments (e.g., Domjan et al., 1992), presumably to decrease the likelihood of the birds' 
fear responses to novel environments. In this particular experiment, it may also have resulted in decreased contextual excitation via latent inhibition and thereby increased the modulatory properties of each context.

All of the subjects were given equal amounts of exposure to the sand and wire contexts throughout the experiment, and the context assignment was counterbalanced. Subjects received either a CXT+ trial or a CXT - trial each day (because of the alternate housing procedure). During training trials, copulatory opportunity with a female quail (the US) was provided for male quail in the female's side cage following CS presentation in one of the contexts (CXT + ) but not in the other context (CXT-). In $\mathrm{CXT}+$, each trial began with a 30 -sec pre-CS period. Immediately following the pre-CS period, the CS block was lowered, and $30 \mathrm{sec}$ later, the door to the female's side cage was raised, the male quail entered the side cage, and it was given copulatory opportunity with the female quail for $5 \mathrm{~min}$. In CXT - , following the 30 -sec pre-CS period, the CS block was lowered, and $30 \mathrm{sec}$ later, the door was raised but no female quail was presented. On both CXT + and $\mathrm{CXT}-$ trials, males initially had to be prompted to enter the female's side cage, but after a few trials, the males entered the side cage when the door opened (whether or not a female was present in the side cage). Female quail typically did not enter the male's test cage through the door between the male's test cage and the female's side cage.

All of the subjects received $20 \mathrm{CXT}+$ trials and $20 \mathrm{CXT}-$ trials, one type each day for 40 days.

It is highly unlikely that the presence or absence of the female in the side cage during CS presentation served as an additional discriminative cue for CXT + and CXT - trials. First, the investigators did not observe vocalizations being made by the female quail in the side cages during the conditioning trials. Second, in other sexual conditioning experiments, male quail did not respond differently in conditioned approach responding to a CS when a female bird was housed in a side cage and when she was not (Akins et al., 1994). Similarly, Domjan et al. (1992) did not find differences in conditioned approach behavior toward a holding box based on whether or not a female bird was held there during the trial.

Following discrimination training, all of the subjects were given a preference test. During the preference test, the partition between the two compartments was removed and subjects were placed midway between the two contexts with access to both contexts for $10 \mathrm{~min}$.

\section{Response Measures}

For both CXT + and CXT - trials, the amount of time that subjects spent in the CS zone, a small area marked off around the CS, during the 30 -sec pre-CS period and during the 30 -sec CS period was recorded. The amount of time that subjects spent in each context during the preference test was recorded and the percentage of time that subjects spent in each context was calculated.

Approximately half of the trials were scored from videotapes by a second observer (C.T.C.) who was blind to any experimental manipulations that occurred after the CS presentation. The interobserver reliability correlation was .96 for the amount of time that subjects spent in the CS zone during the $30-\mathrm{sec}$ pre-CS period and .92 for the amount of time that subjects spent in the CS zone during presentation of the CS.

Data for the 20 conditioning trials are presented in 10 blocks of two trials each. The .05 alpha level was used for all statistical decisions.

\section{Results and Discussion}

\section{CS Period}

The mean percentage of time that subjects spent in the CS zone of each context across trial blocks is shown in
Figure 3. Subjects increased the amount of time that they spent near the CS across blocks in CXT + in comparison with CXT - . An ANOVA revealed a significant context $\times$ block interaction $[F(9,99)=6.99]$. Separate one-way ANOVAs for responding during each type of context trial indicated a main effect of trial blocks for CXT + $[F(9,99)=14.1]$, as well as a main effect of trial blocks for CXT - $[F(9,99)=3.84]$. Subsequent simple oneway analyses at each block indicated that the percentage of time that subjects spent near the CS in CXT + was greater than that in CXT- on Blocks $4,7,8,9$, and 10 $[F \mathrm{~s}(1,11)>6.55]$.

A significant main effect of context indicated that, overall, subjects spent more time near the CS in CXT $+(M=$ $56.54 \%, S E M=2.64 \%)$ than in CXT $-(M=42.95 \%$, $S E M=2.82 \%)[F(1,11)=17.92]$. There was also a significant main effect of block $[F(9,99)=8.9]$.

\section{CS-Pre-CS Difference Scores}

To aid in determining the degree of excitation acquired by the context during CXT + and CXT - trials, CS - preCS difference scores were computed for each subject by subtracting pre-CS scores from CS scores. If subjects spent similar amounts of time in the zone during the preCS period (in the absence of the CS) as they did during the CS period, difference scores should be close to zero and the increase in approach behavior that was evident across trial blocks in the CS data should not be evident in the difference score data. A two-factor ANOVA with context and block as variables was performed on the difference scores. The results of the analysis were similar to the results from the CS period data (above). The results showed that subjects increased time spent in the CS zone during the CS across trial blocks when they were in the positive context but not when they were in the negative context. Means $( \pm S E M)$ ranged from $17.07 \%( \pm 6.35 \%)$

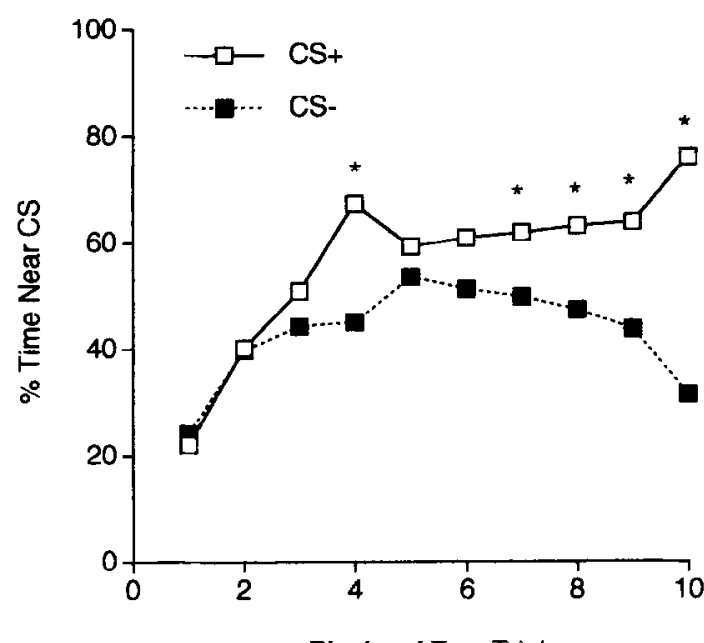

Blocks of Two Trials

Figure 3. Mean percentage of time that subjects spent in the CS zone during discrimination training as a function of trial blocks. 
on Block 1 to $64.46 \%( \pm 7.42 \%)$ on Block 10 for the positive context and from $17.29 \%( \pm 7.76 \%)$ on Block 1 to $45.38 \%( \pm 8.71 \%)$ on Block 5 . This was indicated by a significant context $\times$ block interaction $[F(9,99)=$ 4.67]. There were also significant main effects of block $[F(9,99)=3.7]$ and context $[F(1,11)=6.14]$. Thus, context did not appear to be excitatory in the absence of the CS.

\section{Preference Test}

At the end of training, subjects were given access to $\mathrm{CXT}+$ and to CXT-, and the amount of time that they spent in each context was recorded. Subjects spent $67.11 \%$ $( \pm 11.47 \%)$ of their time in CXT + and $32.89 \%( \pm 11.47 \%)$ in CXT - . A one-factor ANOVA with CXT as a variable did not reach statistical significance $[F(1,11)=2.23]$. One possible explanation for this result is that contextual excitation was subthreshold and thereby insufficient to elicit a behavioral preference. Another possibility is that context did not acquire excitation but rather served to modulate responding to the discrete CS in the absence of contextual excitation.

\section{EXPERIMENT 3}

The findings of Experiment 2 support the previous finding that context may facilitate conditioned responding to localized stimuli. They further suggest that contextual excitation of sexual conditioning may not always accompany modulatory control of conditioned sexual behavior. In Experiment 2, the US was presented in a context other than the context that had been trained as the modulator. This may have weakened any excitatory association between the context and the US, and may subsequently have allowed for the enhancement of the modulatory control of the context.

Evidence from previous research suggests that experimental procedures may determine whether or not the context comes to control responding of CSs by simple associations or by its occasion-setting ability independently of direct associations with a US. Ross and Holland (1981) found that the temporal relationship between a feature and target stimulus determined the mechanism used to control responding. If the temporal relationship was close (simultaneous), the feature stimulus developed control of responding through its excitatory association with the US. However, when the temporal relationship was not close (serial), the feature acquired the ability to "set the occasion."

The purpose of Experiment 3 was to vary the "spatial contiguity" of the context and US to determine whether or not greater spatial distance between the context and US would alter the context-US association and thus influence the function of context. We compared sexually conditioned approach in two groups of subjects that received discrimination training. While in $\mathrm{CXT}+$, one group of subjects received the US in the same context as that in which the discrete CS was presented (Group Same), whereas the other group received the US in the female's side cage (Group Alternate). Presentation of the CS in CXT - was the same for both groups. Just as in the previous experiment, the US was copulatory opportunity with a female quail, and time spent in the CS zone was used as an index of sexually conditioned approach behavior. To assess contextual excitation, three context preference tests were given: one prior to training, one in the middle of training, and one at the end of training.

\section{Method}

\section{Subjects}

Twelve male Japanese quail, approximately 5-12 months old, served in Experiment 3 . They had been raised and housed under the same conditions as in Experiments 1 and 2. An equal number of female quail was used during the periods of copulatory opportunity. The assignment of females to males was rotated across trials, as in Experiments 1 and 2.

\section{Apparatus}

The same experimental chambers were used as in Experiment 2.

\section{Procedure}

Experiment 3 was conducted in two replications of 6 subjects each. During each replication and prior to any experimental manipulations, half of the subjects were placed in the sand context and half were placed in the wire context. Thereafter, they were moved from one context to the other at around noon each day, ensuring that all of the subjects had equal amounts of exposure to each context for the duration of the experiment. This context exchange procedure began 10-14 days prior to the start of the experiment. We avoided using the alternate housing procedure that was used in Experiment 2, because we wanted to ensure against generalization or interference of the colony cages with the test contexts.

Prior to training, subjects were randomly assigned to one of two groups, Group Same or Group Alternate. The subjects in Group Same received copulatory opportunity with a female quail (the US) in the same context as that in which the CS was presented during CXT + training. In contrast, the subjects in Group Alternate received the US in an alternate context from that in which the CS was presented during CXT + training.

Training trials were conducted as in Experiment 2, except that on each day subjects received a CXT + trial and a CXT - trial. Following a $30-$ sec pre-CS period and a $30-$ sec CS presentation, a US was either presented or not. During CXT + triais, for Group Same, a female bird that was housed in the side cage was removed from the side cage and introduced through the front door of the test chamber. Male quail were given $5 \mathrm{~min}$ to copulate with her in the test chamber (the US). For the subjects in Group Alternate, during $\mathrm{CXT}+$ trials, male quail had to enter the side cage to gain access to a female quail and copulate with her there. Any differences in responding could not be attributed to the location of the female quail during the CS presentation, because the female quail were always housed in the side cage during CS presentation in CXT + . During CXT - trials, Group Same received the 30 -sec CS block followed by opening of the front door but no presentation of a female bird. Group Alternate received the 30-sec CS block followed by opening of the side door and no female quail presentation. Just as in Experiment 2, the subjects in Group Alternate were initially 
prompted through the side cage when the door opened during $\mathrm{CXT}+$ and CXT - trials. All of the subjects received $20 \mathrm{CXT}+$ and $20 \mathrm{CXT}$ - training trials, one of each per day.

The subjects were given an initial preference test prior to training. Subsequent preference tests were conducted after every 10 th $\mathrm{CXT}+/ \mathrm{CXT}-$ training day. Preference tests were conducted by removing the partition between the two contexts, placing male quail midway between the two contexts, and recording the amount of time that subjects spent in each context for $10 \mathrm{~min}$. Three preference tests were conducted.

\section{Response Measures}

During training, the amount of time that subjects spent in the CS zone was recorded during the 30 -sec pre-CS and the 30 -sec CS periods. The amount of time that subjects spent in each context was recorded during the preference tests. This was calculated into the percentage of time that subjects spent in each context.

Approximately half of the training trials were scored from videotapes by a second observer who was blind to the subjects' group assignments. The interobserver reliability correlation was .90 for the amount of time that subjects spent in the CS zone during the $30-$ sec pre-CS period prior to $\mathrm{CS}$ exposure. The interobserver reliability correlation was .89 for the amount of time that subjects spent in the CS zone during presentation of the CS.

All of the preference tests were videotaped and scored by a second observer, and the interobserver reliability correlation was .94 for the amount of time that subjects spent in each context.

Data for the 20 training trials are presented in 10 blocks of two trials each. The .05 alpha level was used for all statistical decisions.

\section{CS Period}

\section{Results and Discussion}

The mean percentage of time that Groups Same and Alternate spent in the CS zone across CXT + and CXTtrial blocks is shown in the top panel of Figure 4. The spatial contiguity of the context and US following CXT + trials had a great impact on the amount of time that subjects spent near the CS across CXT+ and CXT - trials. This was represented as a significant three-way interaction of block $\times$ context $\times$ group $[F(9,90)=2.25]$. A separate two-factor analysis for Group Same indicated that the subjects that received the US in the same context as they received the CS did not differ in responding to the CS across $\mathrm{CXT}+$ trials as opposed to CXT - trials $[F(9,45)=1.48]$. In contrast, the subjects in Group Alternate, which received the US in a context alternate to that in which they received the $\mathrm{CS}$, responded to the $\mathrm{CS}$ in $\mathrm{CXT}+$ significantly differently across trials than they did in CXT$[F(9,45)=7.12]$.

The three-factor ANOVA also revealed a significant main effect of block $[F(9,90)=5.63]$, a significant main effect of context $[F(1,10)=41.74]$, and a significant context $\times$ block interaction $[F(9,90)=4.61]$.

\section{CS-Pre-CS Difference Scores}

As in Experiment 2, we wanted to use another measure to aid in determining the degree of contextual excitation acquired by CXT + and CXT - . CS - pre-CS difference scores were computed for the percentage of time in the CS zone. An ANOVA with context, block, and group served as variables. The results showed that, col-
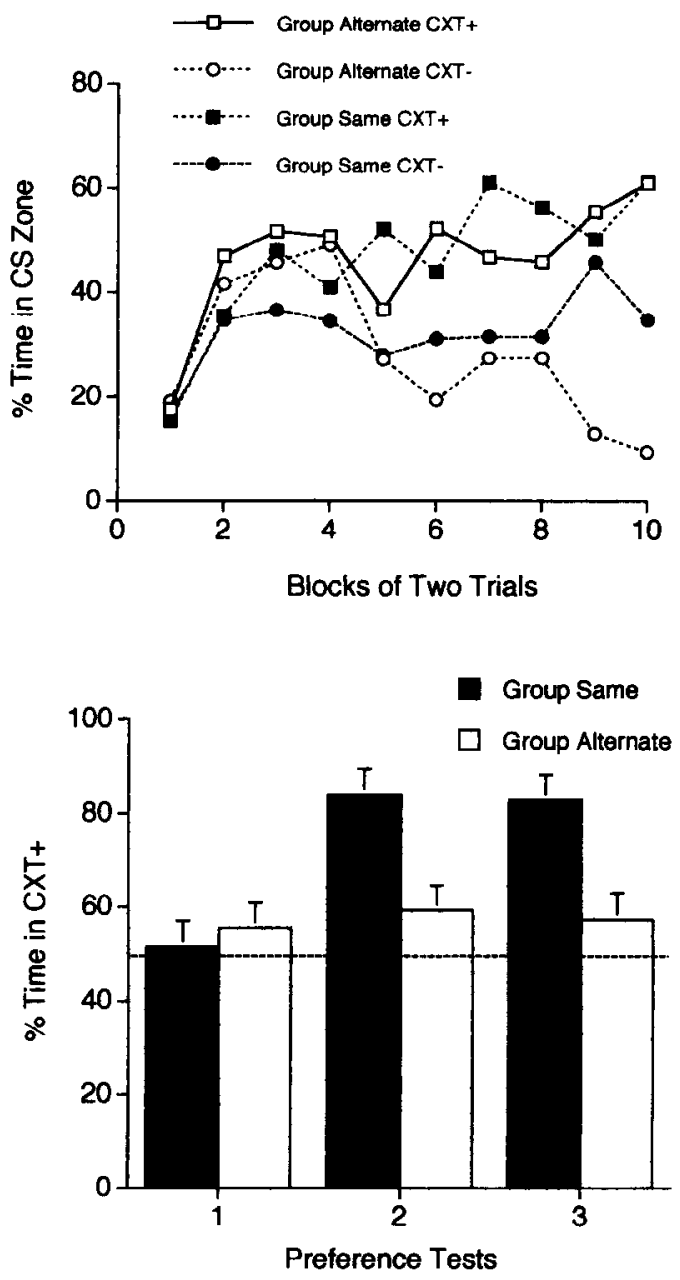

Figure 4. Mean percentage of time that Group Same and Group Alternate spent in the CS zone during discrimination training as a function of trial blocks (top panel). The bottom panel represents the mean percentage of time that subjects spent in the context where the CS was followed by a US.

lapsed across group, $\mathrm{CS}-$ pre-CS difference scores were similar to the CS period results. Subjects increased the percentage of time that they spent in the CS zone during $\mathrm{CXT}+$ trials but not during $\mathrm{CXT}-$. This was indicated by a significant context $\times$ block interaction $[F(9,90)=$ 3.74]. There were also significant main effects of block $[F(9,90)=2.02]$ and context $[F(1,10)=28.92]$ and a significant three-way interaction of context, block, and group $[F(9,90)=2.05]$. Separate one-way ANOVAs were performed to identify the source of this interaction. During CXT + trials, Group Alternate increased its time spent in the CS zone across trials $[F(9,45)=2.65]$, whereas Group Same did not $[F(9,45)=2.05]$. This differs from what was found in the CS period results, which suggests that both groups increased in approach behavior during CXT + trials. In contrast, the analysis with 
CS-pre-CS difference scores indicated that subjects that received the US in the same context responded similarly during the pre-CS period and during CS presentation. This provides evidence for the acquisition of contextual excitation in subjects that received the US directly in the context. Results for the CXT - trials were similar for CS-pre-CS difference scores and for the CS period data.

\section{Preference Test}

The bottom panel of Figure 4 represents the mean percentage of time that Group Same and Group Alternate spent in the CXT+ during each of the three preference tests. Group Same increased the amount of time that they spent in CXT + across preference tests, whereas Group Alternate did not. An ANOVA revealed a significant group $\times$ test interaction $[F(2,20)=6.78]$. Overall, the subjects in Group Same spent more time in CXT + than did the subjects in Group Alternate. Means were $72.7 \%$ $( \pm 4.73 \%)$ and $57.4 \%( \pm 2.98 \%)[F(1,10)=7.12]$. In addition, the main effect of test was significant $[F(2,20)=$ 9.68].

During preference tests between the $\mathrm{CXT}+$ and $\mathrm{CXT}-$, male quail preferred the CXT + over the CXT -, but only when a female quail had been released in the $\mathrm{CXT}+$. When a female quail was released in an alternate context, male quail did not show a preference for the CXT + over the CXT - . Rather, they spent about half of their time in each context.

\section{GENERAL DISCUSSION}

In the present series of experiments, the mechanism of context in sexual conditioning was investigated by (1) directly pairing context with a US (Experiment 1), (2) assessing contextual excitation after explicitly training context to modify responding to a discrete CS (Experiment 2), and (3) manipulating the spatial relationship between the context and the US in the presence of a discrete CS (Experiment 3). Male Japanese quail served as subjects and access to a sexually receptive female quail served as the US. Context preference tests were used to assess the acquisition of contextual excitation. During discrimination training, the amount of time that subjects spent in a small area that contained the CS was recorded as an index of conditioned approach behavior. In addition, approach to the area was monitored during a pre-CS period, prior to CS presentation. CS - pre-CS difference scores were calculated to further assess the degree of contextual control.

In the absence of a discrete stimulus, a context that was directly paired with a US acquired excitatory properties. Male Japanese quail shifted their preference from an initially preferred familiar context to one in which they received copulatory opportunity with a female bird. In addition, male quail showed an increase in locomotor activity in the US-paired context across conditioning trials. This contextual cue excitation occurred after only five context-US pairings and was extinguished after five extinction trials in which the US was no longer presented after context exposure. These findings are consistent with other sexual conditioning studies that have provided evidence for the acquisition of a direct association of context and a sexual US (e.g., Zamble et al., 1985).

Experiment 2 provided evidence for modulatory effects of context on sexually conditioned responding to a discrete CS. These findings replicate and extend the findings of Domjan et al. (1992). Male quail demonstrated increased conditioned approach behavior to a CS when it was followed by a US in one context but not when it was presented in an alternate context followed by no US. In addition, the context preference test following modulatory training of the contexts indicated that the context may not have acquired excitation. One possible explanation for this finding is that the context-US associations were subthreshold and therefore did not elicit a behavioral context preference. Alternatively, contextual excitation may not have accompanied the context's modulatory role of the discrete CS on sexual conditioning. A third possibility is that the context did not acquire an association with the US because of the place in which the US was presented. In Experiment 1, there was a context preference for a place in which male quail were allowed to copulate with a female quail. In Experiment 2, however, male quail copulated with the female quail in a place other than the putative modulatory context. Therefore, context was not spatially paired with the US during modulatory training in Experiment 2. This may have strengthened the modulatory control of the context and weakened the acquisition of context excitation.

In Experiment 3, the "spatial contiguity" of the context and the US were manipulated. The context was trained as a discriminative cue such that in one context, the CS was followed by the US (the positive context), whereas in another context (the negative context), the CS was followed by no US. Presentation of the US directly in the positive context following CS presentation resulted in the acquisition of contextual excitation. This was manifest as a context preference and as similar approach behavior to the CS zone during the pre-CS period and during the CS period. In contrast, presentation of the US in an alternate context following CS presentation failed to result in contextual excitation. There was no preference for one context over the other during preference tests, and subjects showed approach behavior to the CS area only during CS presentation. Although many other factors may influence contextual control of sexual conditioning, our findings suggest that the spatial contiguity between the context and the US may play a role in determining whether or not context excitation accompanies modulatory control of sexual conditioning. Close spatial contiguity between a context and a US resulted in the context's serving a modulatory role but also acquiring excitation. In contrast, when spatial contiguity between the context and the US was greater, the context served to modulate conditioned sexual behavior but it did not appear to ac- 
quire excitation. Thus, there may be a functional dichotomy between simple sexual conditioning and the modulatory contextual control of sexual conditioning that is a function of the spatial relationship between the context and the US.

The findings provide evidence that context excitation may be an integral part of the modulatory control of sexual conditioning. The available research with discrete stimuli as modulators in other preparations indicate that excitation is not a component of modulation (see Swartzentruber, 1995, for a review). Similarly, studies involving the manipulation of context excitation and modulatory control of fear conditioning have demonstrated that contexts control responding to discrete CSs in the absence of demonstrable excitation or inhibition with US associations (e.g., Bouton \& King, 1983, 1986; Bouton \& Swartzentruber, 1986; Swartzentruber \& Bouton, 1988).

It should be noted that we cannot definitively argue that the context served as an occasion setter or modulator in any of the present experiments. A series of more complicated experiments involving such procedures as transfer tests (e.g., Holland, 1989; Holland \& Reeve, 1991 ) would be necessary to demonstrate occasion setting. Moreover, our findings easily lend themselves to a purely associative learning interpretation. In a hierarchical situation in which a CS, US, and context are present, the acquisition of associative strength between the context and the US competes with the acquisition of associative strength between the CS and the US. The stronger the context-US association, the weaker the CS-US association. By altering the spatial contiguity between the context and the US, we have altered the strength of the context-US association and thereby the strength of the CS-US association. When spatial contiguity between the context and the US was close, the excitatory value of the context was increased. As a result, the context acquired more associative strength than did the CS, and subjects demonstrated a preference for the context. CS-pre-CS difference scores revealed that the context acquired excitation because subjects responded in the absence of the CS. Alternatively, when spatial contiguity between the context and the US was greater, the excitatory value of the context was decreased, the context competed less with the CS for associative strength, responding was specific to the CS, and subjects appeared to be responding to the CS without utilizing information about the context.

Regardless of the interpretation, the present findings provide important and novel information about contextual control of the sexual behavior system. Domjan's (1994) formulation of a sexual behavior system provides an elaborate framework for organizing the effects of various stimuli on conditioned sexual behavior. The present findings contribute to our understanding of the organizing effects of context in conditioned sexual behavior, and they set the stage for future investigations of context manipulations in sexual conditioning experiments. It is clear from our results that simple contextual excitation plays a large role in the contextual control of conditioned sexual behavior. It is further evident that the acquisition of contextual excitation depends on the spatial relationship between the context and the sexual event. It may also be that the modulatory role of the context in controlling sexual responding is influenced by context-US spatial contiguity.

Finally, although the present preference test results have been discussed from the perspective that context acquired excitatory properties if subjects preferred that context, it should be noted that the preference tests were always between the positive and negative contexts. Therefore, preference for a positive context over a negative context may have been due to contextual excitation of the positive context, contextual inhibition of the negative context, or a combination of the two. Moreover, there was no test for contextual inhibition in any of the present experiments.

\section{REFERENCES}

AKIns, C. K., Domjan, M., \& Gutiérrez, G. (1994). Topography of sexually conditioned behavior in male Japanese quail (Coturnix japonica) depends on the CS-US interval. Journal of Experimental Psychology: Animal Behavior Processes, 20, 199-209.

Balaz, M. A., Capra, S., Hartl, P., \& Miller, R. R. (1981). Contextual potentiation of acquired behavior after devaluing direct contextUS associations. Learning \& Motivation, 12, 383-397.

Bolles, R. C., \& Collier, A. C. (1976). The effect of predictive cues on freezing in rats. Animal Learning \& Behavior, 4, 6-8.

Bouton, M. E. (1984). Differential control by context in the inflation and reinstatement paradigms. Journal of Experimental Psychology: Animal Behavior Processes, 10, 56-74.

Bouton, M. E., \& Bolles, R. C. (1985). Contexts, event-memories, and extinction. In P. D. Balsam \& A. Tomie (Eds.), Context and learning (pp. 133-166). Hillsdale, NJ: Erlbaum.

Bouton, M. E., \& KING, D. A. (1983). Contextual control of the extinction of conditioned fear: Tests for the associative value of the context. Journal of Experimental Psychology: Animal Behavior Processes, 9, 248-265.

Bouton, M. E., \& KING, D. A. (1986). Effect of context on performance to conditioned stimuli with mixed histories of reinforcement and nonreinforcement. Journal of Experimental Psychology: Animal Behavior Processes, 12, 4-15.

Bouton, M. E., \& Swartzentruber, D. (1986). Analysis of the associative and occasion-setting properties of contexts participating in a Pavlovian discrimination. Journal of Experimental Psychology: Animal Behavior Processes, 12, 333-350.

Colwill, R. M., Absher, A., \& Roberts, M. L. (1988). Context-US learning in Aplysia californica. Journal of Neuroscience, $\mathbf{8 , 4 4 3 4 -}$ 4439.

Domjan, M. (1994). Formulation of a behavior system for sexual conditioning. Psychonomic Bulletin \& Review, 1, 421-428.

Domjan, M., Akins, C. [K.], \& VANDERGRIFF, D. H. (1992). Increased responding to female stimuli as a result of sexual experience: Tests of mechanisms of learning. Quarterly Joumal of Experimental Psychology, 45B, 139-157.

Domuan, M., Greene, P., \& North, N. C. (1989). Contextual conditioning and the control of copulatory behavior by species-specific sign stimuli in male Japanese quail. Journal of Experimental Psychology: Animal Behavior Processes, 15, 147-153.

Domuan, M., O'Vary, D., \& Greene, P. (1988). Conditioning of appetitive and consummatory sexual behavior in male Japanese quail Journal of the Experimental Analysis of Behavior, 50, 505-519. 
Durlach, P. J. (1982, April). Direct measurement of context conditioning in the pigeon. Paper presented at the 53rd Annual Meeting of the Eastern Psychological Association, Baltimore.

FANSELOW, M. S. (1990). Factors governing one-trial contextual conditioning. Animal Learning \& Behavior, 18, 264-270.

Grahame, N. J., Hallam, S. C., Geier, L., \& Miller, R. R. (1990). Context as an occasion setter following either CS acquisition and extinction or CS acquisition alone. Learning \& Motivation, 21, 237-265.

Hilliard, S., NguYen, M., \& Domuan, M. (1997). One-trial appetitive conditioning in the sexual behavior system. Psychonomic Bulletin \& Review, 4, 237-241.

Holland, P. C. (1983). "Occasion-setting" in conditional discriminations. In M. Commons, R. Hernstein, \& A. R. Wagner (Eds.), Quantitative analyses of behavior: Vol. 4. Discrimination processes (pp. 183206). New York: Ballinger.

Holland, P. C. (1989). Acquisition and transfer of conditional discrimination performance. Journal of Experimental Psychology: Animal Behavior Processes, 15, 154-165.

Holland, P. C. (1992). Occasion setting in Pavlovian conditioning. In D. L. Medin (Ed.), The psychology of learning and motivation (Vol. 28, pp. 69-125). San Diego: Academic Press.

Holland, P. C., \& REEve, C. E. (1991). Acquisition and transfer of control by an ambiguous cue. Animal Learning \& Behavior, 19, 113-124.

MarLIN, N. A. (1981). Contextual associations in trace conditioning. Animal Learning \& Behavior, 9, 519-523.

Mustaca, A. E., Gabelli, F., Papini, M. R., \& Balsam, P. (1991). The effects of varying the interreinforcement interval on appetitive contextual conditioning. Animal Learning \& Behavior, 19, 125-138.

ODLING-SMEE, F. J. (1975). The role of background stimuli during Pavlovian conditioning. Quarterly Journal of Experimental Psychology, 27, 201-209.

ODLING-SMEE, F. J. (1978). The overshadowing of background stimuli by an informative CS in aversive Pavlovian conditioning with rats. Animal Learning \& Behavior, 6, 43-51.

Papini, M. R., Mustaca, A. E., Tiscornia, G., \& DiTella, M. (1987). Contextual learning in the marsupial (Lutreolina Crassicaudata Red Opossum). International Journal of Comparative Psychology, 1 , 126-134.
Rescorla, R. A., Durlach, P. J., \& Grau, J. W. (1985). Contextual learning in Pavlovian conditioning. In P. D. Balsam \& A. Tomie (Eds.), Context and learning (pp. 23-56). Hillsdale, NJ: Erlbaum.

RESCORLA, R. A., \& WAGNER, A. R. (1972). A theory of Pavlovian conditioning: Variations in the effectiveness of reinforcement and nonreinforcement. In A. H. Black \& W. F. Prokasy (Eds.), Classical conditioning II: Current research and theory (pp. 64-99). New York: Appleton-Century-Crofts.

Roald Maes, J. H., \& Vossen, J. M. H. (1993). Context conditioning: Positive reinforcing effects of various food-related stimuli. Physiology \& Behavior, 53, 1227-1229.

Ross, R. T., \& Holland, P. C. (1981). Conditioning of simultaneous and serial feature-positive discriminations. Animal Behavior \& Learning, 9, 293-303.

Schein, M. W., Diamond, M., \& Carter, C. S. (1972). Sexual performance levels of male Japanese quail (Coturnix coturnix japonica). Animal Behaviour, 20, 61-67.

SWARTZENTRUBER, D. (1991). Blocking between occasion setters and contextual stimuli. Journal of Experimental Psychology: Animal Behavior Processes, 17, 163-173.

Swartzentruber, D. (1995). Modulatory mechanisms in Pavlovian conditioning. Animal Learning \& Behavior, 23, 123-143.

Swartzentruber, D., \& Bouton, M. E. (1988). Transfer of positive contextual control across different conditioned stimuli. Bulletin of the Psychonomic Society, 26, 569-572.

Zamble, E., Hadad, G. M., Mitchell, J. B., \& Cutmore, T. R. (1985). Pavlovian conditioning of sexual arousal: First- and second-order effects. Journal of Experimental Psychology: Animal Behavior Processes, 11, 598-610.

Zamble, E., Mitchell, J. B., \& Findlay, H. (1986). Pavlovian conditioning of sexual arousal: Parametric and background manipulations. Journal of Experimental Psychology: Animal Behavior Processes, 12, 403-411.

(Manuscript received March 2, 1998; revision accepted for publication July 19, 1998.) 